\title{
GIGASPERMUM MOURETII CORB. EN LAS ISLAS BALEARES
}

\author{
Juan RITA \& José A. ROSSELLO
}

\begin{abstract}
RESUMEN; En el presente trabajo se cita por primera vez en Baleares a Gigaspermum mouretii Corb., hasta el momento estas islas representan el límite septentrional del área de distribución de esta especie. Se presenta una descripción detallada del esporófito de la misma, así como algunos datos acerca del sustrato donde vive, que sugieren una preferencia por suelos descarbonatados y con contenidos relativamente elevados en nutrientes.
\end{abstract}

Palabras clave: Gigaspermum, Musci, corología, ecología, Islas Baleares.

ABSTRACT: We quote Gigaspermum mouretii Corb. form the first time in Balearic Island. To this moment these island represent the northern limit in the distribution area for this species. We also present a detailed description of its sporophyte, as well as some data concerning the substrate where it lives, suggesting a preference for decarbonated soils with a relatively high nutrient content.

Key words: Gigaspermum, Musci, chorology, ecology, Balearic Islands.

Durante unas herborizaciones realizadas en el Sur de Mallorca se detectó un pequeño musgo acrocárpico de coloración amarillenta y con abundantes esporófitos que formaba extensos céspedes en los claros del matorral esclerófilo de esta zona. Posteriormente fue encontrado en hábitats similares del Sur de la isla de Formentera.

La consulta de diversas floras briológicas europeas (Augier, 1966; Nyholm, 1954; Smith, 1978) para su identificación, no nos permitió llegar a ningún resultado. Una parte del material fue enviado a M. Brugués (Barcelona) quien lo determinó como Gigaspermum mouretii Corb.

Este taxon era conocido con anterioridad del Norte de Africa, de donde fue descrito originalmente (Corbiere, 1913), de algunas localidades del Sureste de la Península Ibérica (Allorge \& Casas, 1958; Casas, 1974; Acuña et al., 1974; Ros, 1984) y de Israel (Herrnstadt et al., 1980). Las plantas procedentes de Israel fueron recolectadas con esporófitos, desconocidos hasta entonces, y a pesar de que fueron inconografiados (Herrnstadt et al., 1980) no se describieron en el texto.

Ya que las características del esporófito de esta especie no han sido dadas a conocer, creemos oportuno presentar en esta nota sus características principales, así como esbozar algunos aspectos del hábitat de la misma, con especial atención a sus preferencias edáficas. El gametófito ha sido descrito con detalle por Ros (1984). 


\section{DE S C R IP CION}

Hojas perigonales no diferenciadas, hojas periqueciales (Fig. 1 y 2) de mayor tamaño, agudas y apiculadas, con los bordes de los ápices, a veces, involutos. En algunas hojas periqueciales aparece una doble capa de células en su parte central, que puede alcanzar los $2 / 3$ del mismo y que se puede considerar como una nerviación rudimentaria (Fig. 2, c).

Paroico. Cápsula ovoide, subsesil (seta de hasta $60 \mu \mathrm{m}$ ), atenuada en la base, de 1-1.3 x 0.9-1.2 mm. Opérculo cónico, mamilado, peristoma nulo. Células de la boca de la urna cuadradas o subrectangulares, incrasadas de 10-28 x 14-42 $\mu \mathrm{m}$, dispuestas en 2-3 hileras, las demás poligonales de 40-60 x 16-45 $\mu \mathrm{m}$. Estomas inmersos localizados en la base de la urna (Fig. 3).

Esporas poligonales, de color castaño, de hasta $130 \mu \mathrm{m}$ de diámetro, sin abertura visible. Perina débilmente papilosa observada al M.O. Al SEM se observan macrogránulos verruculiformes, variables en tamaño y coalescentes en algunos casos; superficie con microgránulos aparentemente lisos, dispuestos irregularmente y dejando microper-foraciones entre ellos (Lám. 1).

\section{DISTRIBUCION, CLIMA Y FENOLOGIA}

Hemos hallado a G. mouretii en las siguientes localidades del Sureste de Mallorca: S'Aranjassa (31SDE78), y Marina de Llucmajor (31SDE58, 31SDE68) y del extremo Sur de Formentera: Cap de Barbaria (31SCC75) (Fig. 4). Los pliegos testimonio se encuentran depositados en el herbario de la Universitat de les Illes Balears (UIB).

Todas estas localidades (Fig. 4) se encuentran a una altitud inferior a $200 \mathrm{~m}$ s.n.m., bajo unas temperaturas y precipitaciones medias que oscilan entre $16.3 \mathrm{y}$ $17.3^{\circ} \mathrm{C}$ y 350 y $390 \mathrm{~m}$ en las localidades mallorquinas y de $17.8^{\circ} \mathrm{C}$ y $380 \mathrm{~mm}$ en Formentera (Guijarro, 1986). Todas ellas se pueden encuadrar en el Piso Termomediterráneo con ombroclima seco o semiárido (Rivas-Martínez, 1983).

Se ha observado anteridios y arquegonios en el mes de Noviembre. El desarrollo de la cápsula se produce en los meses de Enero y Febrero, mientras que la esporosis se inicia en Febrero y Marzo.

\section{CARACTERISTICAS DEL SUSTRATO}

El sustrato litológico del territorio donde se ha encontrado Gigaspermum mouretii está formado en su totalidad por rocas calcáreas, que en su mayor parte son eolianitas pleistocénicas y, en menor medida, calcarenitas miocénicas.

Gigaspermum mouretii ha aparecido colonizando suelos esqueléticos y litosuelos, en ocasiones en contacto con la roca aflorante. Estos suelos pueden clasificarse dentro del subgrupo Litic xerorthents.

Para conocer sus preferencias edáficas se han analizado siete muestras de suelos colonizados por esta especie. Las muestras abarcaron la totalidad del perfil, puesto 

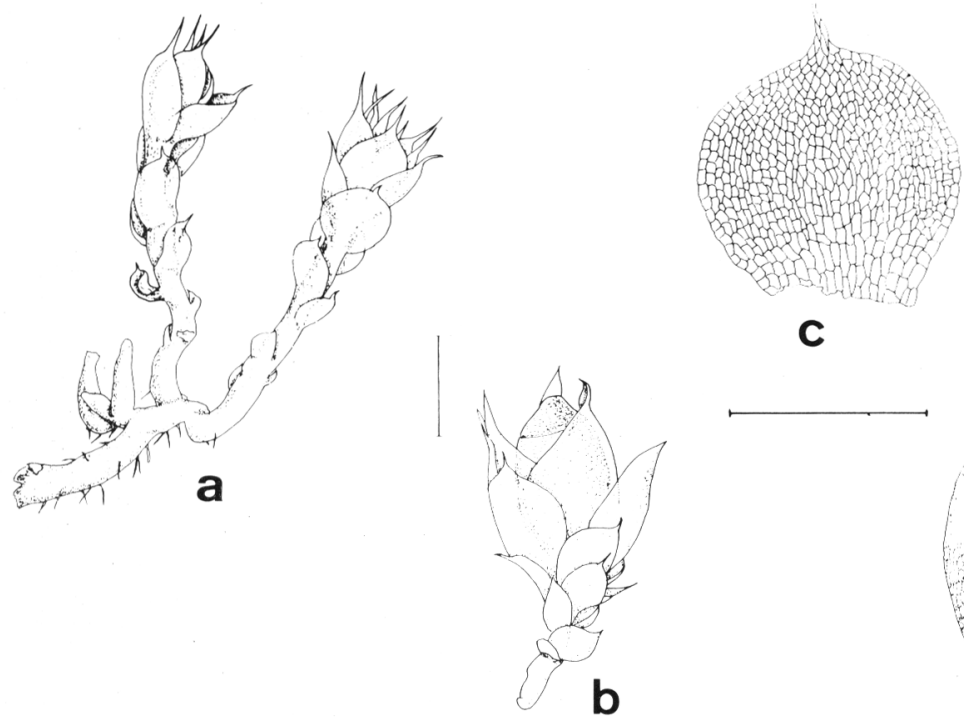

Fig. 1: a.- Detalle de los tallos del gametófito; b.- Parte apical de un tallo fértil; c.Areolación de un filidio caulinar; d.- Esporangio. Escala a, b, c, d: $1 \mathrm{~mm}$.

Arec
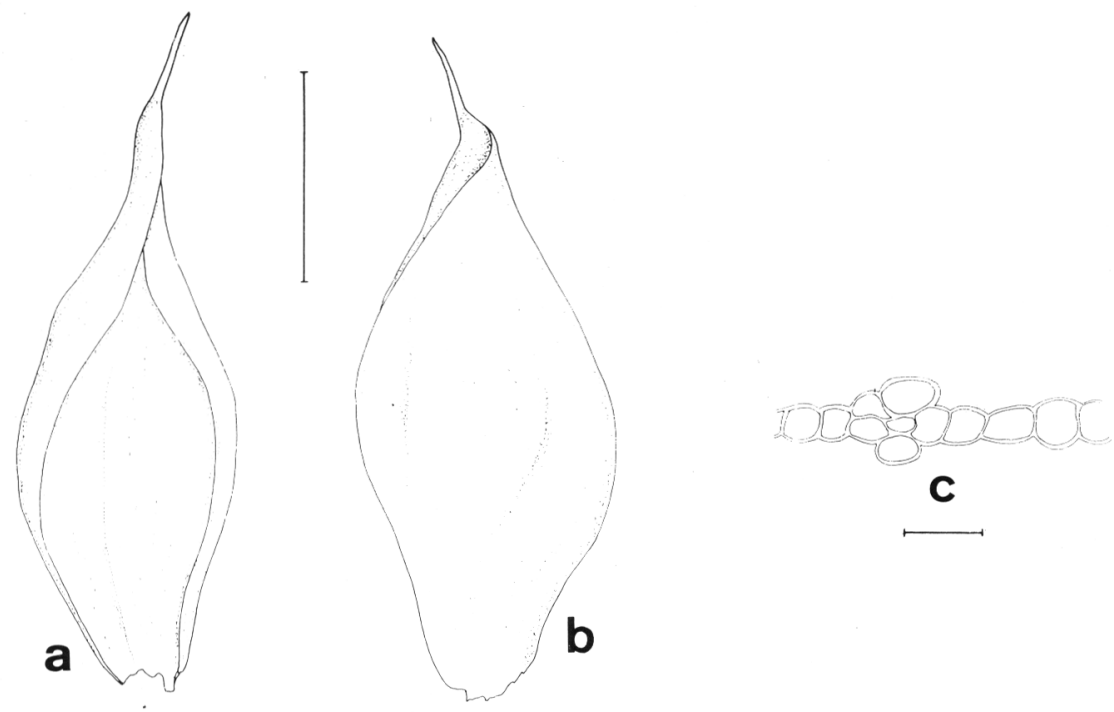

C

Fig. 2: a, b.- Filidios periqueciales; c.- Sección transversal de la parte media. Escala a, b: $500 \mu \mathrm{m}$; c: $50 \mu \mathrm{m}$. 

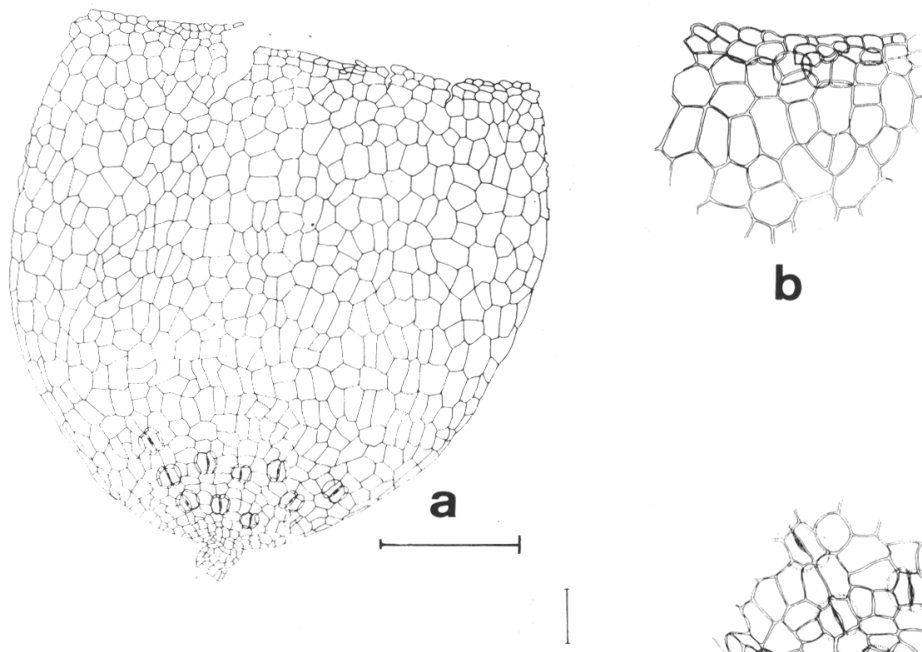

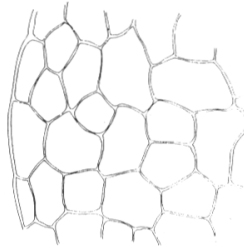

C

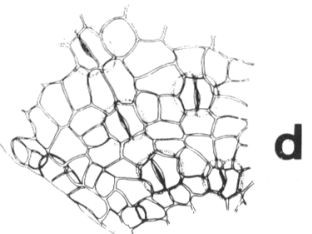

Fig. 3: Cápsula. (a) Areolación celular y (b) detalle de la parte superior (c) media e (d) inferior de la misma. Escala a: $250 \mu \mathrm{m}, \mathrm{b}, \mathrm{c}, \mathrm{d}: 50 \mu \mathrm{m}$.

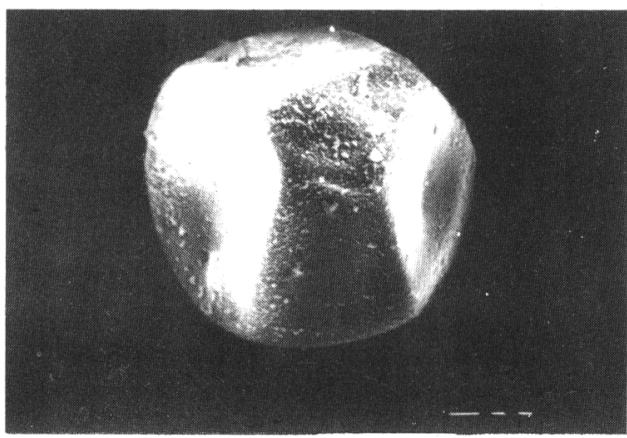

a
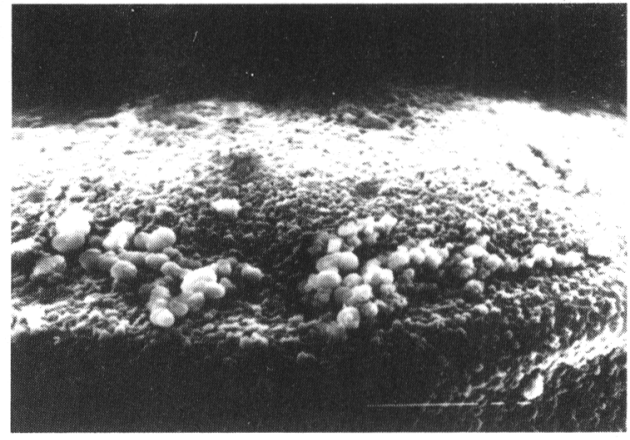

b

Lám. 1: a.- Espora, b.- Detalle de la perina. Escala a: $20 \mu \mathrm{m}$; b: $10 \mu \mathrm{m}$. 
que la profundidad de los mismos no sobrepasó los $15 \mathrm{~cm}$. De estas muestras se desecharon los 5-10 $\mathrm{mm}$ superficiales.

En la tabla $n^{2}$. 1 se detallan las medias, desviaciones típicas y valores extremos de las variables edafológicas que fueron analizadas. Los métodos de análisis fueron los habituales en edafología.

Tabla 1.

\begin{tabular}{lrrrr}
\hline \hline & Media & Desv. tip. & Maxim. & Minim \\
\hline Grava \% & 0.8 & 2.0 & 5.0 & 0.0 \\
Ar. gruesa \% & 0.9 & 0.6 & 2.0 & 0.4 \\
Ar. fina \% & 51.6 & 17.0 & 68.7 & 30.5 \\
Limo \% & 26.9 & 7.8 & 39.1 & 18.5 \\
Arcilla \% & 20.6 & 11.2 & 39.5 & 12.0 \\
pH & 7.6 & 0.4 & 8.1 & 7.2 \\
Donduc. $\mu$ S/cm & 179.0 & 71.0 & 266.0 & 85.0 \\
$\mathrm{CaCO}_{3} \%$ & 0.1 & 0.3 & 0.8 & 0.0 \\
$\mathrm{Cl}^{-}$meq/1 & 0.5 & 0.2 & 0.9 & 0.3 \\
$\mathrm{Na}^{+}$ppm & 239.0 & 35.0 & 288.0 & 188.0 \\
$\mathrm{~K}^{+}$ppm & 250.0 & 69.0 & 36.0 & 18.0 \\
$\mathrm{Ca}^{++}$ppm & 4928.0 & 1068.0 & 6220.0 & 3918.0 \\
$\mathrm{Mg}{ }^{++}$ppm & 293.0 & 77.0 & 401.0 & 171.0 \\
$\mathrm{~N} \%$ & 0.36 & 0.11 & 0.56 & 0.23 \\
$\mathrm{Mat}$. Org. $\%$ & 6.4 & 2.3 & 9.6 & 3.7 \\
$\mathrm{C} / \mathrm{N}$ & 9.6 & 0.4 & 10.0 & 9.2 \\
P ppm & 12.0 & 4.0 & 18.0 & 5.0 \\
\hline
\end{tabular}

Una de las características más destacables de estos suelos es que su contenido en carbonatos es tan bajo que ha resultado indetectable por nuestro método de análisis (Calcímetro de Bernard). En la zona Sur de Mallorca los suelos sin carbonatos no son raros, pero coexisten con otros con más de un $25 \%$ de $\mathrm{CaCO} 3$. Es frecuente que ambos tipos de suelos aparezcan en las mismas localidades (Rita, 1988).

En esta región la ausencia de carbonatos suele estar asociada a contenidos bajos en arenas gruesas $(2000-200 \mu \mathrm{m})$ y de gravas $(>2 \mathrm{~mm})$. Efectivamente ambas fracciones representan menos del $1 \%$ como valor medio de nuestras muestras.

Dado el número de muestras analizadas aún es prematuro adjudicar a esta especie el calificativo de calcífuga, aunque es muy probable que tenga una marcada preferencia por los suelos sin carbonatos y débilmente alcalinos ( $\mathrm{pH}$ medio de $7.6 \pm$ 0.4 medido en solución acuosa 1/2.5).

El contenido en materia orgánica es relativamente elevado $(6.43 \pm 2.27 \%)$ dado que se trata de muestras superficiales.

La proporción de fósforo asimilable (método Olsen) también es bastante elevada $(12.4 \pm 4.5 \mathrm{ppm})$, ya que se encuentra dentro del rango superior del conjunto de suelos no agrícolas de esta zona de Mallorca. El potasio intercambiable presenta valores muy heterogéneos, aunque el valor medio también se encuentra en los niveles superiores de los suelos de este territorio. La coincidencia de valores relativamente altos en estas tres últimas variables (materia orgánica, fósforo asimilable y potasio 
intercambiable) sugiere que Gigaspermum mouretii presenta una tendencia a vivir sobre suelos ricos en nutrientes.
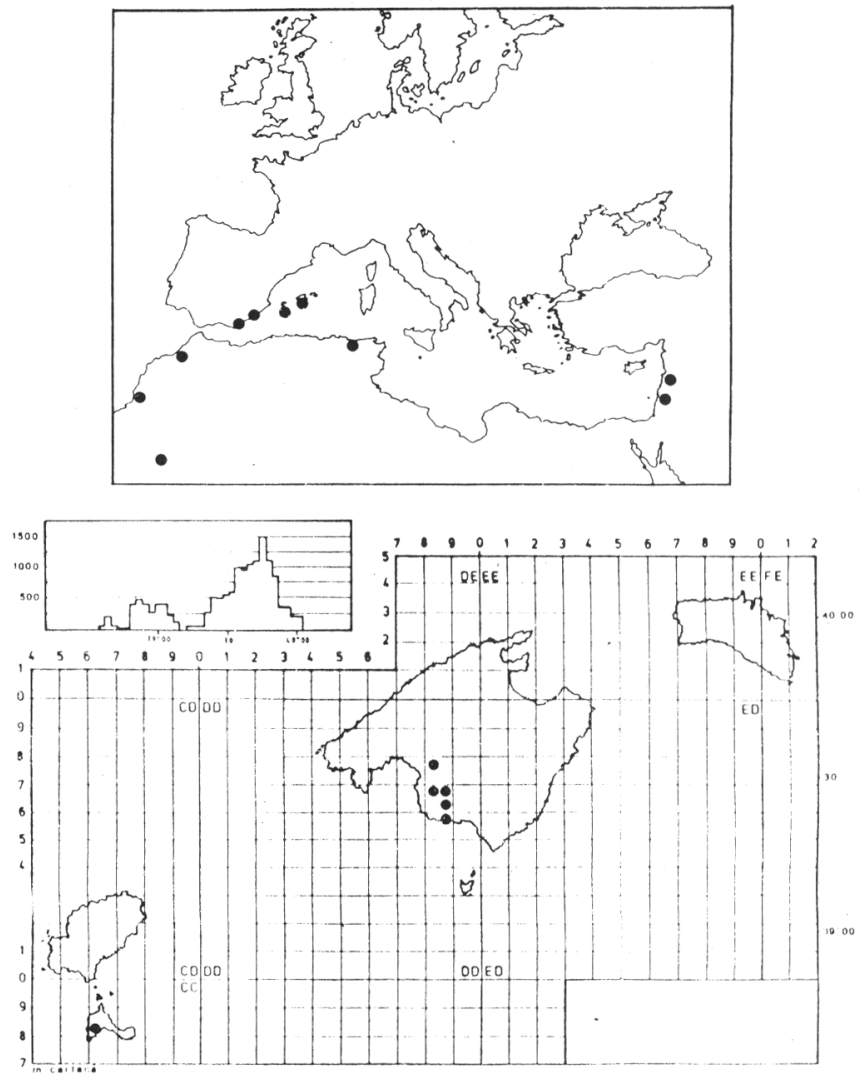

Fig. 4. Distribución conocida de Gigaspermum mouretii en Baleares y en el resto de su areal.

\section{FLORA ACOMPAÑANTE}

La vegetación leñosa predominante es un matorral alto pero abierto formado por especies esclerófilas entre las que dominan Olea europaea y Pistacia lentiscus que ha sido adscrita a la As. Cneoro-Ceratonietum (Al. Oleo-Ceratonion) (Bolòs \& Molinier, 1958). En los claros de este matorral vive Gigaspermum mouretii acompañado por una comunidad muy rica en terófitos y briófitos efímeros $(\mathrm{Cl}$. Thero-Brachypodietea y Cl. Barbuletea unguiculatae). Las especies de briófitos que con mayor frecuencia acompañan a $G$. mouretii son: Riccia lamellosa, $R$. papilosa, $R$. nigrella, $R$. gougetiana, Oxymitria paleacea, Fossombronia caespitiformis, Petalophyllum ralfsii, Weissia ssp., Bryum ssp., Pottia spp., Trichostomum spp., etc. Las fanerógamas acompañantes más comunes son Crassula tillaea, Sedum rubens, Sagina apetala, 
Trifolium suffocatum, Bellis annua, y ocasionalmente Gymnostyles stolonifera y Crepis pusilla.

\section{DIS CUSION}

La presencia de G. mouretii en Mallorca y Formentera supone, hasta el momento, el límite septentrional de su área de distribución. Su porte insconspícuo, y las probables confusiones sobre el terreno con formas de Bryum argenteum, permiten suponer que esta especie pueda tener una distribución en el Mediterráneo mucho más amplia de la que se conoce en la actualidad (Fig. 4).

El sustrato sobre el que se desarrolla G. mouretii tal como han indicado autores anteriores, junto con nuestras propias observaciones sugieren que se trata de un taxon calcífugo, si bien sobre suelos neutros o ligeramente básicos.

La ausencia o rareza de esporófitos en esta especie (siendo un taxon monoico) podría atribuierse a diferentes factores:

1.- El período en que fueron llevadas a cabo las herborizaciones fuera anterior a la fecundación del arquegonio, con lo que únicamente aparecerían indivíduos con gametangios.

2.- Las escasas precipitaciones y el régimen irregular de las mismas en las localidades donde vive $G$. mouretii, puede restringir o anular la fertilidad de sus poblaciones (tal como sugiere Herrnstadt et al. (1980); sin embargo, la frecuencia del rocío en estas zonas litorales puede aportar el agua necesaria para que se puede producir la fecundación; además la formación de céspedes, típica de esta especie, debe favorecer la acumulación de agua proveniente de esta via aneja.

3.- Finalmente podría argüirse que dado que la fragmentación del rizoma subterráneo de G. mouretii es un excelente mecanismo de reproducción asexual, podría darse una reducción del potencial reproductor sexual. Sin embargo, la abundante producción de gametangios en las poblaciones ibéricas y baleáricas examinadas (pese a que Acuña et al. (1974) y Ros (1984) señalan que las poblaciones ibéricas son estériles), junto con las referencias proporcionadas por Corbiere (1913) y Herrnstadt et al. (1980) parecen apuntar que no es éste el caso.

\section{BIBLIOGR AFIA}

ACUÑA, A., CASAS, C., COSTA, M., FUERTES, E., LADERO, M., LOPEZ, M.L., SIMO, R.M., VARO, J. -1974- Aportaciones al conocimiento de la flora briológica española. Nótula I: El Cabo de Gata (Almería). Anal. Inst. Bot. Cavanilles 31(2):59-95.

ALLORGE, V., CASAS, C. -1958- Contribution à la flore bryologique de l'Espagne. Rev. Bryol. Licheinlol 27:55-65.

BOLOS, O. DE, MOLINER, R. -1958- Recherches phytosociologiques dans l'île de Majorque. Coll. Bot., 5:699-865.

CORBIERE, L. -1913- Contribution à la flore bryologique du Maroc d'apres les récolectes du lieutenant Mouret. Rev. Bryol. 40(1):7-13.

CASAS, C. -1974- Quelques muscinées de la terre du Cabo de Gata et leur relation avec la flore bryologique africane. Bull. Soc. Bot. France, coll. Bryologie 121:313-318.

GUIJARRO, J.A. -1986- Contribución a la bioclimatología de Baleares. Tesis Doctoral, 
Universitat de les Illes Balears, Palma de Mallorca.

HERRNSTADT, I, CHAIA, C.H., CROSBY, M.R. -1980- New data on the Moss Genus Gigaspermum. The Bryologist 83(4):536-541.

RIVAS MARTINEZ, S. -1983- Pisos bioclimáticos de España. Lazaroa 5:33-43,

RITA, J. -1988- Estructura y ecología de los pastizales terofíticos de Baleares. El medio y la vegetación de la Marina de Llucmajor. Tesis Doctoral. Universidat de les Illes Balears.

ROS, R.M. -1984- Una nueva localidad de Gigaspermum mouretii Corb. (Musci) en la Península Ibérica. Acta Bot. Mal. 9:93-96.

(Aceptado para su publicación el 16 de enero de 1989)

Dirección de los autores: Laboratorio de Botànica. Departament de Biologia i C de la S. Universitat de les Illes Balears. Palma de Mallorca. 